\title{
La juventud en el contexto de la estructura social cubana. $D$ atos y reflexiones
}

\author{
M aría I sabel D omínguez G arcía \\ Centro de Investigaciones Psicológicas y Sociológicas (CIPS). \\ D epartamento de Estructura y Política Social. \\ Calle B, \#352, esquina a 15. 10400 Vedado. La H abana. Cuba
}

\section{Resumen}

La juventud constituye uno de los grupos más significativos de la sociedad cubana por su alto peso numérico, sus actuales rasgos cualitativos y una larga tradición de protagonismo social a lo largo de distintas etapas de la historia nacional.

El artículo analiza al sector juvenil en el contexto de la estructura generacional, demográfica y socioclasista de la población y aborda los cambios que se están produciendo en su situación social como efecto de la crisis económica que vive el país desde 1990 y de la estra tegia de reestructuración que se desarrolla para salir de ella.

Palabras clave: juventud, estructura social, generaciones, inserción social de la juventud.

\begin{abstract}
The youth in the social structure of Cuba
Youth is one of the key social groups of Cuban society because of the number of young people and their traditional role in Cuban history.

The article analyses the changes in the social situation of this group after the economic crises since 1990 and the implementation of restructuring policies in Cuba.
\end{abstract}

Key words: youth, social structure, youth social insertion.

\section{Sumario}

Introducción La juventud en la estructura

Algunas precisiones conceptuales

La juventud en la estructura generacional de la población

La juventud en la estructura ocupacional

Cambios en la inserción de la juventud en la estructura socioclasista demográfica A modo de conclusión 


\section{Introducción}

Las reflexiones sobre juventud y su papel en la sociedad cuentan con una amplia tradición en el pensamiento social cubano, por tratarse de una población con un fuerte peso de sus sectores juveniles y porque éstos han actuado como protagonistas principales de nuestra historia en sus diferentes etapas.

A su vez, la importancia que el proyecto de la Revolución concedió a las nuevas generaciones y su educación, condicionó la temprana aparición de dispositivos institucionales para analizar el tema y el inicio de los estudios empíricos sobre la juventud antes que muchos otros de relevancia social ${ }^{1}$.

La crisis económica que atraviesa el país desde 1990 - la mayor que haya sufrido a lo largo de todo el siglo- , cuyos efectos se dejan sentir sobre toda la vida de la sociedad y sobre cada uno de los grupos que la conforman, así como la estrategia de reestructuración que se lleva a cabo para salir de ella, impactan a la juventud con mayor intensidad que a otras generaciones por el momento de la vida en que se encuentran, tanto en términos sociales como psicológicos.

Ello coloca al grupo juvenil en el centro de atención de las investigaciones sociales, y una de las aristas más significativas del análisis es su lugar en la estructura social de la sociedad.

\section{Algunas precisiones conceptuales}

El significado de la categoría «juventud» ha atravesado por diferentes niveles de desarrollo, desde el definido sólo por límites de edades derivados por los cambios biológicos o a lo sumo psicológicos, hasta la inclusión de los criterios sociales como los decisivos en su determinación. D e igual forma ha ocurrido con la aceptación del carácter histórico concreto del concepto, que implica la particular delimitación de la edad juvenil en cada sociedad y la necesaria renovación de esos límites en correspondencia con los cambios que se operan en la vida real.

En nuestros estudios partimos de una concepción de juventud en la que ésta se define como una categoría histórico-concreta que designa un grupo sociodemográfico internamente diferenciado según su pertenencia a la estructura social de la sociedad, en particular a las distintas clases y capas que la componen, a la vez que constituyen su segmento más dinámico y móvil. Enfatizamos que la juventud no está biológicamente determinada, sino definida socialmente por la naturaleza de la actividad que se desarrolla en esa etapa, la que condiciona un conjunto de relaciones sociales específicas que conforman el status juvenil a partir del significado propio de dicho período. Esto constituye una identidad juvenil, que es tanto autoidentidad como identidad reconocida por el resto de las generaciones?

1. Domínguez, M .I. «Las investigaciones sobre la juventud en Cuba». Revista Tema, núm. 1, enero-marzo de 1995. La H abana, p. 85-93.

2. D o M ÍN GUEZ, M .I . «C riterios teórico-metodológicos para la investigación de la juventud». Revista cubana de Ciencias Sociales, núm. 17, año VI, mayo-agosto de 1988. La H abana. 
Esta concepción de juventud, aun cuando admite su carácter como etapa del ciclo de vida y tiene que recurrir al establecimiento de límites de edades para poder operacionalizarla, pone el énfasis en las relaciones sociales de que es portador el grupo en su conjunto, así como cada una de sus subdivisiones, ya sean etéreas o de otra natural eza. Por tal razón, no es posible su estudio sin ubicarla en el contexto de la estructura social en la que se inserta y sin considerar, sobre todo, su doble pertenencia a la estructura generacional de la pobla ción y a la estructura socioclasista.

A tal efecto partimos de definir conceptualmente a la generación como: «El conjunto histórico concreto de personas, próximas por la edad y socializadas en un determinado momento del proceso histórico del país, lo que condiciona una actividad social común en etapas claves de formación de la personalidad, que da lugar a rasgos estructurales y subjetivos similares, que la dotan de una fisonomía propia»3.

Esta definición - aunque coincide con la mayor parte de las que han sido elaboradas a lo largo de la historia del concepto, al destacar que es un grupo de personas que vive y se forma en una determinada etapa histórica- pone el énfasis en el carácter de la actividad social desplegada colectivamente como lo realmente conformador de la generación. Es por esta razón que, a diferencia de los principales teóricos que han definido las generaciones y han usado el método generacional para analizar procesos sociales concretos, no encuadramos cada una de ellas en interval os fijos y regulares, sino por el contrario en períodos variables según los vaivenes del movimiento real de la historia ${ }^{4}$.

A su vez, esta manera de concebir las generaciones obliga a no desconectar sus análisis del estudio de la estructura socioclasista, es decir, del conjunto de clases, capas y grupos sociales que se derivan de la división social del trabajo y de las relaciones de propiedad imperantes. D esde este punto de vista, generaciones y clases sociales constituyen eslabones de una enmarañada red de relaciones.

3. Ibídem.

4. Generalmente, las concepciones sobre generaciones a lo largo de la historia han pretendido fijar la duración de cada una de ellas en interval os fijos. Así la duración de cada una se ha definido según los principales ciclos de la vida, la esperanza de vida u otros criterios. Por ejemplo, la primera formulación conocida, hecha por Thomas J efferson en 1789, las consideraba en ciclos de 19 años; las propuestas positivistas derivadas de la concepción de A. Comte como la de J. D rommel (1862) o F. M entré (1920) que consideraron ciclos de 16 años y 30 años respectivamente, o las más elaborada de José 0 rtega y $\mathrm{G}$ asset, asumida también por Julián M arías, que concibió cinco generaciones de 15 años de extensión.

Un análisis más detallado puede consultarse en D o m ín GUEZ, M .I. «U n recorrido histórico por el tratamiento de las categorías generacionales, juventud y socialización». En Las generaciones y la juventud: una reflexión sobre la sociedad cubana actual. CIPS/1994 (en proceso de publicación). 


\section{La juventud en la estructura generacional de la población}

En Cuba actualmente coexisten cuatro grupos generacionales, integrados por las personas que constituyen grupos de edades significativos y que ya han podido conformarse como tales, lo que abarca a los nacidos entre 1922 y 1975. Para su composición ${ }^{5}$ ver la tabla 1.

Aunque el nivel de agrupación por edades que brindan las estadísticas oficiales no permite su ajuste exacto a las generaciones, una visión aproximada de las magnitudes de cada grupo está reflejado por la tabla 2.

Los cuatro grupos generacionales que hoy son contemporáneos, han acumulado una experiencia vital heterogénea en dependencia de sus vínculos con los procesos sociales de cambio, de los que sin duda alguna el más intenso fue el iniciado con el triunfo de la Revolución. Ello condiciona los rasgos más relevantes de cada uno de los grupos y da lugar a un cuadro generacional que puede resumirse de la siguiente manera ${ }^{6}$ :

1. U na generación mayor ${ }^{7}$, socializada totalmente en la etapa pre-revolucionaria con una larga trayectoria de intensa actividad social en las esferas del trabajo, de la defensa del país y en el terreno sociopolítico, que incluye a los propios protagonistas de la R evolución, representante de una fuerte movilidad geográfica y social, y con niveles de instrucción y calificación inferiores al de las generaciones subsiguientes.

2. Un grupo intermedio (los nacidos entre 1944 y 1949), muy pequeño pero muy peculiar, que ha representado un grupo generacional de transición entre los mayores y las generaciones que le sucedieron. Esta generación también ha desplegado una intensa actividad social, y en ocasiones ha concentrado los niveles más altos de participación, con la particularidad de que ésta se ha mantenido de manera constante a lo largo de distintas etapas de su vida y de la Revolución. Este grupo constituye el máximo expo-

5. La investigación sobre la estructura generacional de la población cubana actual partió de una periodización histórica del contexto en que socializaron las personas mayores de 14 años que hoy conforman la población, a partir de sus puntos de cambio más significativos. Esa periodización dio origen a la hipótesis del cuadro generacional que debía existir. La hipótesis se sometió a comprobación empírica con una muestra representativa de la población cubana por clases y capas sociales. Los resultados obtenidos permitieron precisar los verdaderos límites de cada generación, obligó a recortar unas y alargar otras respecto de la propuesta inicial, para quedar conformada de la forma que se señala.

D o m ín GUEZ, M .I. Estructura Generacional dela población cubana actual. CIPS, 1989. Informe de investigación.

6. Esta síntesis se apoya en los resultados de la investigación Las generaciones en la sociedad cubana actual. D omínguez, M .I.; M .E. Ferrer; M.V. Valdés. CIPS. La H abana, 1990. Informe.

7. En realidad, aquí se agrupan dos generaciones, cuya línea demarcatoria la constituye los nacidos entre 1932 y 1936, pero por la fuerte similitud que existe entre ellos es posible considerarla conjuntamente y su distinción sólo es necesaria para análisis muy específicos. 
Tabla 1. Estructura generacional de la población cubana.

\begin{tabular}{lll}
\hline Primera generación: & N acidos entre 1922 y 1943 & Entre 52 y 73 años \\
Segunda generación: & $N$ acidos entre 1944 y 1949 & Entre 46 y 51 años \\
Tercera generación: & $N$ acidos entre 1950 y 1961 & Entre 34 y 45 años \\
Cuarta generación: & $N$ acidos entre 1962 y 1975 & Entre 20 y 33 años
\end{tabular}

Fuente: D o mínguez, M .I. Estructura generacional de la población cubana actual (informe de investigación). CIPS, 1989.

Tabla 2. Proporción de población aproximada por generaciones.

\begin{tabular}{lc} 
Generaciones & $\%$ \\
\hline Primera & 28,2 \\
Segunda & 7,6 \\
Tercera & 27,7 \\
Cuarta & 36,5 \\
Total & 100
\end{tabular}

Fuente: Calculado a partir de Anuario D emográfico de Cuba, 1993. ComitéEstatal de Estadística. Tabla I.14, p. 123.

nente de la movilidad social intergeneracional ascendente, en tanto por su edad tuvo la posibilidad de usufructuar desde el inicio las oportunidades educativas creadas en la década de 1960 y con esa mayor preparación satisfacer las nuevas demandas ocupacionales que se abrían.

Esta generación constituye también un grupo de transición en términos de valores y normas de conducta en esferas vinculadas a la familia, las relaciones de pareja, los roles entre los sexos, las relaciones raciales, etc., que posteriormente se consolidaron en los más jóvenes, pero que iniciaron su transformación en ella. Con todos estos cambios el grupo protagonizó un primer momento de ruptura generacional en relación con sus mayores.

3. Dos generaciones más jóvenes, nacidos entre 1950 y 1975, socializadas completamente en la etapa revolucionaria, que representan al rededor de las dos terceras partes del total de población adulta, fuertemente urbanizadas portadoras de los más altos niveles de instrucción y calificación alcanzados por grupo alguno, pero con un ritmo menor de movimiento de ascenso social y con una inferior participación social que sus predecesores, excepto en la esfera educacional. Ambas generaciones son portadoras de cambios en los patrones de conducta vinculados a la vida familiar, entre los que se destaca un elevado peso de uniones consensuales, un menor números de hijos (un hijo) y una reducción de la edad de inicio de la maternidad.

Aunque estos rasgos caracterizan a las dos generaciones, todas las tendencias enunciadas se dan con mayor agudeza en la menor. 
Es necesario enfatizar que si bien este es el cuadro más global a nivel de toda la población, las características generacionales se hallan matizadas por la pertenencia a uno u otro componente de la estructura socioclasista, pues el peso fundamental aun recae sobre las clases o capas sociales, mientras que las diferencias generacionales están presentes, principalmente, en el interior de cada componente.

N o obstante, como los efectos de la crisis y los reajustes son vividos de manera desigual por las distintas generaciones, y en el caso de los jóvenes de manera más intensa, por los límites que impone a su inclusión actual y a su proyección de futuro, se puede apreciar entre ellos una cierta consolidación generacional. N o quiere esto decir que desaparezcan las distinciones entre grupos sociales y clasistas, incluso se refuerza la heterogeneización de algunas actitudes juveniles, pero aparecen tendencias que apuntan hacia la conformación de una identidad juvenil integrada y relativamente diferenciada de las generaciones anteriores que potencian en estos momentos las peculiaridades generacionales más que en otras etapas.

A este proceso se incorporan los menores de 20 años, que no se incluían en la estructura anterior, porque es precisamente ahora cuando se están conformando como generación: La generación de los noventa. De los rasgos que asuma la juventud actual podrá definirse con mayor precisión el límite inferior de la cuarta generación y el alcance que tiene la que está surgiendo. H abría entonces que preguntarse ¿quiénes son los jóvenes de hoy?

\section{La juventud en la estructura demográfica}

En Cuba se ha establecido la etapa de la juventud entre los 14 y los 30 años, pues se ha considerado que en nuestras condiciones en esa etapa se produce el proceso de maduración del individuo, tanto desde el punto de vista biológico, como psicológico y social y, por tanto, a ese sector de la población van dirigidas las políticas de juventud. Sin embargo, se toma en cuenta que entre esas edades hay notables diferencias que permiten distinguir tres subgrupos:

- La juventud temprana (14 a 17 años).

- La juventud media (18 a 24 años).

- La juventud madura o tardía (25 a 30 años)8.

La composición actual de la juventud - tanto su magnitud como su estructura interna- es el resultado de cambios demográficos significativos que tuvieron lugar en el país en las últimas cuatro décadas, sobre todo el incremento explosivo que tuvo la natalidad entre 1959 y 1964 y que mantuvo altas tasas hasta 1972, seguido de un decrecimiento brusco de los nacimientos que se

8. Dom ínguez, M .I. «Criterios teóricos metodológicos... ». Artículo citado. 
Tabla 3. D istribución de la juventud cubana por subgrupos de edades. Año 1994.

\begin{tabular}{ccc} 
Grupos de edades & Valores absolutos & $\%$ \\
\hline Total 15-29 años & 3.152 .302 & 100 \\
15-19 años & 852.513 & 27 \\
20-24 años & 1.134 .547 & 36 \\
25-29 años & 1.165 .242 & 37
\end{tabular}

Fuente: Datos del Comité ESTATAL DE ESTADíst ICA del 1 de enero de 1994.

conserva hasta hoy ${ }^{9}$. Ello dio lugar a una estructura de edades en la población caracterizada por un abultamiento que ha transitado por la pirámide de edades para situarse, desde la segunda mitad de los años ochenta, en la edad juvenil, lo que hace que la sociedad cubana sea eminentemente joven.

Aunque a partir de 1990 se inicia un decrecimiento del número absoluto de jóvenes, en 1994 existían en el país 3.152.302, que representaban el 28,8 \% del total de la población ${ }^{10}$. Si añadimos el grupo infantil (2.433.396), la proporción de personas menores de 30 años alcanza el 52,2\%. Este el evado peso se mantendrá hasta el año 2000, en que los adultos comenzarán a ser mayoría. Para esa fecha 721.000 personas habrán dejado de ser jóvenes ${ }^{11}$.

Según las proyecciones real izadas, la estructura interna de la juventud continuará modificándose hacia un predominio del grupo de más edad, mientras el sector menor ha decrecido significativamente: En 1995 se había reducido en casi medio millón respecto de 1987. A partir de ahora se iniciará una lenta recuperación de este último segmento, que hará que al iniciarse el nuevo siglo al cance una proporción dentro del total de jóvenes similar a la que representaba en $1990^{12}$.

Este proceso general tiene fuertes variaciones territoriales (por provincias del país). Baste decir que mientras en Ciudad de La H abana, que concentra alrededor del $20 \%$ de los jóvenes cubanos, predomina desde 1991 el grupo tardío, en el resto de las provincias ello no ocurrirá hasta 1996-199713.

Los cambios en la estructura interna tiene repercusiones sobre el tipo de demandas fundamentales que el grupo juvenil plantea a la sociedad, pues han pasado de las educacionales y recreativas que fueron las mayoritarias en etapas anteriores, a las de empleo, vivienda, atención a sus hijos y participación social.

Del total de jóvenes el $50,7 \%$ son del sexo masculino y el $49,3 \%$ del femenino ${ }^{14}$.

9. Álvarez, L. La fecundidad en Cuba. Editorial Ciencias Sociales. La H avana.

10. Fuente: COM ITÉ ESTATAL DE ESTADístICA. Información sobre población del 1/1/1994.

11. Fuente: Comité Estatal de Estadística. Proyección de indicadores estadísticos seleccionados en relación con la población joven de Cuba hasta el año 2005. La H abana, 1985.

12. I bídem.

13. I bídem.

14. Fuente: Com ité Estatal de Estadística. Anuario demográfico, 1993. Tabla I.14, p. 123. 
O tra característica demográfica del grupo juvenil es su alta concentración urbana, en correspondencia con la transformación de la población en su conjunto, que en 17 años incrementó esa proporción del $60 \%$ al $72 \%{ }^{15}$. En la actualidad el $72 \%$ de los jóvenes vive en las ciudades, con una proporción lige ramente superior de mujeres que de hombres ${ }^{16}$.

U no de los rasgos más significativos de la juventud cubana actual es ser portadora de más elevados niveles de instrucción y calificación que cualquier otra generación: Aunque el promedio de escolaridad se sitúa en el noveno grado, casi la mitad sobrepasa ese nivel, a la vez que el número de jóvenes matriculados en las aulas universitarias ha representado a lo largo de la última década (hasta el curso 1993-1994) al rededor de la quinta parte del total de esas edades ${ }^{17}$.

Aunque uno de los efectos más visibles de la crisis económica y de la estrategia de reestructuración que se sigue para salir de ella, es precisamente la reducción de la matrícula de la enseñanza superior y la ampliación de la enseñanza politécnica de nivel medio, en el último curso escolar estudiaban en las universidades 153.479 jóvenes ${ }^{18}$, que representaban aproximadamente la sexta parte de todos los que se sitúan en el rango de edades comprendido por dicho nivel.

A su vez las acciones educativas han favorecido la calificación profesional de la juventud. En una muestra nacional de personas nacidas entre 1962 y 1975, el 60\% tenía algún tipo de calificación profesional (básica, media o superior) ${ }^{19}$.

En la esfera educacional, vale la pena señalar que se viene produciendo desde hace varios años - y se ha reforzado en los últimos- una tendencia a la feminización de la enseñanza superior. D iferentes factores parecen actuar para conformar la tendencia, que se origina fundamental mente en el mayor rendimiento escolar de las hembras en el nivel medio que les permite ocupar posiciones más favorables en el ordenamiento para ingresar en la universidad. Entre ellos pueden mencionarse desde una maduración (biológica y psicológica) mayor de las hembras en esas edades, hasta - y sobre todo- una socialización con más alto nivel de control familiar, que favorece una mayor aplicación y disciplina ante el estudio y desarrolla el nivel de responsabilidad. Por su parte, la socialización del varón induce la aspiración - y a veces la necesidad realde no postergar demasiado el inicio de la vida laboral y en otros casos, el llamado

15. Fuente: Comité Estatal de Estadística. Anuario Estadístico de Cuba, 1988. Tabla II.3, p. 60.

16. Fuente: Comité Estatal de Estadística. Anuario Demográfico de Cuba, 1993. Tabla I.14, p. 123.

17. Fuente: UNESC O . Anuario Estadístico, 1993. París.

18. Fuente: M INISTERIO DE EDUCACIÓN SUPERIOR. Información sobre matrícula por ramas de la Ciencia (Curso 1985-1986 al 1994-1995). Resumen nacional.

19. D om ínguez, M.I.; Ferrer, M.E.; Valdés, M.V. Las generaciones en Cuba. Informe citado. 
(o la perspectiva de Ilamado) a cumplir el Servicio M ilitar General, interrumpe o desestimula la continuidad de los estudios ${ }^{20}$.

Como resultado en el período 1990-1995, el 57\% de todos los estudiantes universitarios del país son del sexo femenino y no constituyen mayoría sólo en aquellas carreras vinculadas a la tecnología (diferentes ingenierías) y a la rama agropecuaria ${ }^{21}$.

\section{La juventud en la estructura ocupacional}

Uno de los rasgos más distintivos de la juventud cubana de los ochenta, que la diferenció sustancialmente de la de otras regiones y en especial de la de América Latina, fue su fuerte inclusión social, garantizada, sobre todo, por una adecuada inserción laboral. En la segunda mitad de los ochenta llegó a representar el 50\% de la fuerza de trabajo ocupada aun cuando constituía alrededor del $40 \%$ de la población en edad laboral.

Pero esa incorporación al trabajo no sólo fue significativa en el plano cuantitativo, sino también en el cualitativo al concentrarse en actividades de vital importancia, tanto desde el punto de vista económico como social. Por ejemplo, representaban el $32 \%$ del total de obreros industriales, pero constituían entre dos quintas partes y la mitad en ramas clave como la industria electrónica y electrotécnica (48\%), la industria de productos metálicos (43\%), la minería y la metalurgia ferrosa (39\%). D e igual forma representaban el $43 \%$ de los profesionales y técnicos del país y el 34\% de los especialistas de la ciencia22.

Estos procesos fueron expresión del carácter ascendente de la movilidad social intergeneracional, que en términos generales significó el crecimiento de los grupos urbanos, los vinculados a la producción industrial, y a ocupaciones de mayores requerimientos de instrucción, calificación y nivel tecnológico.

20. Téngase en cuenta que en Cuba el Servicio M ilitar $\mathrm{G}$ eneral tiene una duración de dos años y a él pueden incorporarse los jóvenes después de los 17 años. G eneralmente éste se desarrolla en un régimen interno e intensivo que no favorece la continuidad de estudios en esa etapa. No obstante, los jóvenes que ya se encuentran matriculados en las aulas universitarias no son llamados a este régimen, sino que se incorporan por períodos discontinuos al finalizar los cursos escolares y por un período mayor una vez graduados. A su vez, desde hace varios años funciona un sistema de estímul os para aquellos jóvenes que se encuentran cumpliendo el Servicio M ilitar, pero con condiciones para continuar estudios y que se destaquen por su disciplina y responsabilidad, a los cuales se les reduce el período a sólo un año y se les da acceso directo para ingresar en la universidad, pero esto sólo alcanza a un grupo reducido.

21. Fuente: M INISTERIO dE EdUCACIÓN SUPERIOR. Información sobre la matrícula de mujeres por ramas de la Ciencia. (Curso 1985-1986 a 1994-1995). Resumen Nacional.

22. D o mínguez, M.I. Tendencias del desarrollo de la estructura social de la juventud cubana. CIPS. La H abana, 1987. Informe de Investigación. 
Tabla 4. Principales componentes socioclasistas de la juventud cubana. Año 1987.

\begin{tabular}{lrr} 
Componente & \multicolumn{2}{c}{$\%$} \\
\hline Clase obrera & 23,4 & \\
$\quad$ O breros productivos & & 19,2 \\
$\quad$ Trabajadores de servicios & & 4,2 \\
Trabajadores intelectuales & 15,5 & 12,1 \\
$\quad$ Especialistas & & 3,4 \\
$\quad$ Empleados & 2,9 & \\
Campesinos & 0,9 \\
$\quad$ Agricultores individuales & & 2 \\
$\quad$ ooperativistas & 17,6 & \\
Estudiantes & 12 & \\
Amas de casa &
\end{tabular}

Fuente: Com ité Estatal de Estadística. Anuario Estadístico de Cuba, 1988. Tabla II.3, IV.13 y XIII .8 y Encuesta Nacional de 0 cupación, 1987.

La estructura socioclasista (de clases, capas y grupos sociales) de la juventud quedó conformada por los componentes de la tabla $4^{23}$.

C omo rasgo particular de esta estructura se destaca el peso de los ocupados en el sector estatal civil: $38,8 \%$ del total de jóvenes y $47 \%$ de los ocupados en dicho sector, con un ligero predominio en la esfera productiva ( $50 \%$, mientras en la no productiva eran el 45\%), fundamentalmente en los sectores educación, salud, finanzas y administración pública, como resultado del mayor peso de la juventud entre los trabajadores intelectuales que entre la clase obrera ${ }^{24}$.

A estos grupos hay que agregar los jóvenes militares, los desvinculados del estudio y el trabajo y otros grupos de jubilados e incapacitados.

Específicamente en ese momento, el grupo de desvinculados del estudio y el trabajo creció por la conjunción de diferentes factores, en primer lugar, el arribo masivo a la edad laboral de los nacidos durante el boom demográfico de inicio de los sesenta, unido a una menor dinámica de funcionamiento de la economía y a otros factores como la inmovilidad de los trabajadores ocupados, por el débil funcionamiento de los mecanismos de recirculación de la fuerza de trabajo, principalmente los evaluativos. A ello hay que añadir una fuerte selectividad hacia el empleo por parte de los jóvenes al producirse cierto desfase entre las potencialidades educativas y las oportunidades laborales disponibles, que provocó la existencia simultánea de déficits y superávits de fuerza de trabajo joven.

Esto hizo que hacia 1987 hubiera crecido el grupo de jóvenes desvinculados del estudio y el trabajo, hasta representar el $74 \%$ de las personas en edad

23. Estas proporciones son sólo aproximaciones, a partir del cotejo de diversas fuentes, hecho por la autora.

24. Los trabajadores intelectuales jóvenes representaban el 53,2\% del total, mientras entre la clase obrera alcanzaban el 43,5\%. Ver: COM ITÉ EstATAL DE EsTAdíst ICAS. Anuario Estadístico de Cuba, 1988. Tablas IV.13 y IV.14. 
laboral desvinculadas de cualquier actividad productiva ${ }^{25}$, pero la puesta en práctica inmediata de un conjunto de medidas para garantizar empleo juvenil hizo que para 1989 se hubieran reducido esas magnitudes.

Sin embargo, en el área ocupacional se han producido importantes cambios a partir de 1990 como resultado de la crisis y el reajuste económico y ello ha impactado notablemente la estructura socioclasista de la población en su conjunto, pero muy especialmente de la juventud. Los efectos de estos procesos sobre la ocupación de los jóvenes pueden resumirse en cinco grandes direcciones ${ }^{26}$ :

\section{Reducción de la proporción de jóvenes empleados en el sector formal} de la economía y cambios en su estructura de ocupaciones

Esa reducción está ocasionada por el cese de al gunas actividades económicas que ha dado lugar a los Ilamados «trabajadores disponibles», o por el abandono voluntario del puesto de trabajo por desestímulo ante condiciones de trabajo inadecuadas y escaso valor real del salario, frente a otras opciones fuera del sector estatal.

Simultáneamente, dentro del propio sector estatal se han abierto nuevas opciones de empleos vinculados a las líneas de desarrollo propuestas, es decir, turismo, fármacos y producción agropecuaria, aunque con magnitudes diferentes y con exigencias, sobre todo de calificación, también diferentes:

- Un primer grupo es el de los empleos vinculados a actividades científicas y de tecnologías de avanzada, con un peso fuerte de la ingeniería genética y la biotecnología, asociado tanto a la industria farmacéutica como a la producción agropecuaria. Esta es una demanda nueva, cuya magnitud es más bien pequeña y exige muy alta calificación. Se nutre de profesionales y técnicos de la propia actividad científica, la docencia superior, los servicios de salud, así como de jóvenes egresados de la enseñanza superior y técnica especializada.

- El segundo grupo es el de empleos vinculados a la actividad turística que tiene magnitud intermedia, no muy extensa, aunque mucho mayor que el grupo anterior. En esta actividad se mezcla el empleo en el sector propiamente estatal con el de las empresas mixtas, aunque en menor escala también hay un conjunto de nuevos empleos vinculados a corporaciones (estatales, mixtas o privadas) no turísticas.

Este grupo de empleos, también relativamente reciente en la estructura de la fuerza de trabajo, exige determinados requisitos de calificación media o superior, así como de preparación cultural en general. En ella se

25. Domínguez, M .I.; FerRer, M .E.; VALDÉS, M.V. (1990). Características generacionales de los estudiantes y desvinculados del estudio y el trabajo. CIPS. La H abana. Informe de investigación.

26. D o mínguez, M.I.; Ferrer, M .E.(1994). Efectos del Período Especial sobrelosjóvenes. CIPS. La H abana, 1994. Informe de investigación. 
ocupan egresados de la enseñanza politécnica y superior que inician su vida laboral, y trabajadores procedentes de otros sectores y ramas de la economía.

- El tercer grupo es el de los empleos vinculados al sector agropecuario, cuya demanda es masiva y, en sentido general, tiene escasos requisitos de calificación, si se tiene en cuenta que ha sido necesario retornar a una agricultura más tradicional. Se ha nutrido fundamentalmente de la reubicación de trabajadores de otras actividades como la construcción y la industria.

Los dos primeros grupos de empleos se han integrado en una elevada proporción con jóvenes, no así en el tercer caso, pues existe una mayor resistencia entre éstos a ocuparse en las labores agropecuarias.

Aunque éstas son las direcciones principales de los cambios, también se están produciendo otros dentro de una misma actividad económica, sobre todo en la esfera productiva, en función de las posibilidades de producción existentes. M uchos de estos movimientos son hacia puestos de trabajo con menor contenido técnico, con predominio del trabajo físico y menores requerimientos de calificación y en ellos los jóvenes han sido importantes protagonistas por su alto peso en las ramas industriales y sus más altos niveles de calificación.

\section{Incremento de la subocupación}

Éste ha sido el resultado de la estrategia de conservar en lo posible los niveles de ocupación y evitar grandes afectaciones sal ariales para los trabajadores que no pudieran mantenerse en sus puestos. Si bien los datos del subempleo son muy difíciles de cal cular, mucho más para conocer las características particulares de los grupos afectados, sí es posible inferir que ha tenido fuerte repercusión sobre los trabajadores jóvenes, pues el sector más afectado es el industrial, que a su vez concentra altas proporciones de menores de 30 años.

3. Incremento del cooperativismo, vinculado fundamentalmente a la producción agropecuaria con la constitución de las U nidades Básicas de Producción Cooperativa (U BPC)

Aunque en esta nueva forma de organización laboral tampoco hay un predominio juvenil, su presencia es apreciable y adquieren un peso mayor que en las otras formas de producción agropecuaria (agricultores individuales o cooperativas de producción).

\section{Aumento del cuentapropismo, al ampliarse las opciones para}

la realización del trabajo por cuenta propia

Ésta era una opción prácticamente inexistente antes de 1990, limitada a muy escasas actividades económicas y reservada fundamental mente para personas jubiladas. A partir de 1993, con la aprobación de un decreto-ley que autoriza 
el trabajo por cuenta propia, se ha producido un amplio crecimiento del sector (en septiembre de 1995 habían registrados 190.000 trabajadores) ${ }^{27}$, de los cuales, al parecer, una parte considerable son jóvenes ${ }^{28}$.

\section{Incremento de la desvinculación laboral}

Las restricciones del empleo, de una parte, y el desequilibrio financiero existente, de otra ${ }^{29}$, han provocado el aumento del grupo de jóvenes desvinculados del trabajo, algunos de los cuales se han insertado en el sector informal de la economía y se han asociado al trabajo por cuenta propia legal mente establecido, aun cuando no aparezcan registrados como tales ${ }^{30}$, mientras otros buscan sus ingresos por vías ilegales y nutren la economía sumergida.

La magnitud del grupo de desvinculados laborales es difícil de calcular a través de las estadísticas de empleo, pues no aparecen como desempleados, ya que en su mayoría no se encuentran buscando trabajo.

Un segmento particular de estos desvinculados lo constituyen los jóvenes que se han dedicado al trabajo sexual ${ }^{31}$, que si bien es un fenómeno circunscrito a aquellos territorios con polos turísticos fuertes, en esos comienzan a alcanzar cierta magnitud (en especial Ciudad de La H abana y Varadero) y se ha podido constatar que muchos de ellos proceden de otros territorios.

\section{Cambios en la inserción de la juventud en la estructura socioclasista}

Resulta, pues, evidente que todo el conjunto de transformaciones económicas y sociales que se producen aceleradamente en el país y sus efectos sobre las oportunidades de instrucción y calificación y sobre el empleo están dando lugar a profundos cambios en la estructura socioclasista de la juventud. El hecho mismo de que esos procesos están en curso, algunos apenas iniciándose, no nos permite aun mostrar una imagen cuantitativa, sin embargo, a mane ra de resumen podemos enumerar sus principales tendencias:

27. Fuente: Conferencia sobre Economía Cubana dictada por la D ra. Elena Alvárez, D irectora del Instituto Nacional de Investigaciones Económicas, el 20 de septiembre de 1995.

28. No disponemos del dato del número de jóvenes inscritos como trabajadores por cuenta propia, pero se conoce que ha crecido la proporción de personas entre 15 y 49 años y que sólo el $28 \%$ del total son jubilados o incapacitados. Fuente: I bídem.

29. La liquidez alcanzó su momento pico en los primeros meses de 1994 y aunque se logró una considerable reducción como resultado de las medidas adoptadas para el saneamiento de las finanzas internas, en agosto de 1995 aún alcanzaban la cifra de 9.100 millones.

30. Aunque la ley no reconoce la contratación de asalariados por parte de los trabajadores por cuenta propia, en la práctica éstos existen. Están además los ayudantes familiares no remunerados. En estos dos grupos, asociados al sector informal, los jóvenes tienen un peso considerable.

31. En Cuba se les conoce como «jineteras», por sus diferencias con la prostitución «clásica»y quizás para eludir la carga semántica de este término. 
- Aparición de nuevos grupos de trabajadores, en la clase obrera y los trabajadores de servicios vinculados a las corporaciones, empresas mixtas y sector turístico, y en los profesionales y técnicos vinculados a las tecnologías de avanzada.

- Crecimiento del grupo de jóvenes trabajadores por cuenta propia o asociados a dicho sector.

- Crecimiento del grupo de jóvenes desvinculados del estudio por devaluación de la educación como pasaporte para el empleo y un mayor nivel de vida.

- Crecimiento de los grupos desvinculados del trabajo, que incrementan los sectores involucrados en la economía sumergida y otros grupos de conductas desviadas.

- Reforzamiento de la autorreproducción de la intelectualidad y de su feminización por la reducción de las matrículas de enseñanza superior.

- Aumento de la diferenciación social por el acceso de una parte de losjóvenes a la moneda libremente convertible por diversas vías que facilita el acceso a un nivel de vida superior al del resto.

Estas tranformaciones se traducen en cambios en los procesos de movilidad social en dos sentidos:

El primero se refiere a un cambio de signo en los procesos reales de movilidad, en los que se reduce la movilidad ascendente y tienen lugar movimientos descendentes: de la ocupación a la subocupación o la desvinculación laboral; del trabajo intelectual al manual, de ocupaciones de mayor calificación a otras de menor nivel; del trabajo industrial al agropecuario o artesanal.

Estos procesos son tanto intrageneracionales como intergeneracionales, pero en estos últimos es donde se experimentan los mayores descensos, pues no es posible reproducir para la actual generación joven las posiciones que habían alcanzado sus padres como resultado del acelerado ascenso que tuvo lugar durante los años sesenta y setenta.

El segundo se refiere al cambio de criterios en la juventud para evaluar la movilidad social como ascendente, pues van perdiendo fuerza elementos como mayor calificación, contenido intelectual, puestos de dirección, que eran los predominantes en etapas anteriores para ganarlo el de posibilidades de acceso a un mayor nivel de consumo ${ }^{32}$.

En síntesis, la existencia en el momento presente de una triple economía, es decir, la economía tradicional (básicamente estatal), la economía emergente conformada por las asociaciones con capital extranjero, y la economía informal, da lugar a una triple estructura social, que en ocasiones existen paralelamente, pero en general se interpenetran y originan una estructura compleja, diferenciada, que tien de a cierta polarización entre sus grupos extremos.

32. D o mínguez, M .I.; Ferrer, M .E. (1996). Jóvenes Cubanos: Expectativasen los ‘90. Editorial Ciencias Sociales (colección Pinos N uevos). La H abana. 
La mayor parte de los impactos que se están produciendo sobre la estructura socioclasista de la sociedad tienen su mayor peso sobre los jóvenes, si no de forma inmediata al menos perspectivamente.

\section{A modo de conclusión}

Baste sólo añadir que la situación económica y social que se ha creado en Cuba a partir de 1990, ha acercado un tanto la problemática de su juventud a la de otras regiones. Digamos, por ejemplo, el problema del empleo; la incorporación al sector informal de la economía; la restricción de oportunidades para el acceso a la enseñanza superior, los desajustes entre aspiraciones y posibilidades de satisfacerlas, en particular las referidas al consumo material que compulsan a la emigración hacia el norte y la reaparición de fenómenos erradicados hace ya mucho tiempo como la prostitución.

Sin embargo, persisten importantes diferencias con otros países, por ejemplo de nuestra área geográfica, que van desde las altas potencialidades para la inserción social debido a sus elevados niveles de instrucción y calificación, hasta los espacios participativos de que disponen, unido al carácter de las políticas de juventud - a pesar de las limitaciones económicas- y a la existencia de una perspectiva de futuro que se fundamenta en la necesidad de construir una alternativa de desarrollo que permita continuar garantizando, entre otras cosas, la plena inserción social de la juventud. 